ACCEPTED MANUSCRIPT

\title{
v-MOKE magnetometry: a solution for devices with fixed electromagnet
}

To cite this article before publication: Fernando Bonetto et al 2020 Meas. Sci. Technol. in press https://doi.org/10.1088/1361-6501/ab9411

\section{Manuscript version: Accepted Manuscript}

Accepted Manuscript is "the version of the article accepted for publication including all changes made as a result of the peer review process, and which may also include the addition to the article by IOP Publishing of a header, an article ID, a cover sheet and/or an 'Accepted Manuscript' watermark, but excluding any other editing, typesetting or other changes made by IOP Publishing and/or its licensors"

This Accepted Manuscript is @ 2020 IOP Publishing Ltd.

During the embargo period (the 12 month period from the publication of the Version of Record of this article), the Accepted Manuscript is fully protected by copyright and cannot be reused or reposted elsewhere.

As the Version of Record of this article is going to be / has been published on a subscription basis, this Accepted Manuscript is available for reuse under a CC BY-NC-ND 3.0 licence after the 12 month embargo period.

After the embargo period, everyone is permitted to use copy and redistribute this article for non-commercial purposes only, provided that they adhere to all the terms of the licence https://creativecommons.org/licences/by-nc-nd/3.0

Although reasonable endeavours have been taken to obtain all necessary permissions from third parties to include their copyrighted content within this article, their full citation and copyright line may not be present in this Accepted Manuscript version. Before using any content from this article, please refer to the Version of Record on IOPscience once published for full citation and copyright details, as permissions will likely be required. All third party content is fully copyright protected, unless specifically stated otherwise in the figure caption in the Version of Record.

View the article online for updates and enhancements. 


\title{
Technical Design Note
}

\section{v-MOKE magnetometry: a solution for devices} with fixed electromagnet

\author{
F. Bonetto ${ }^{1,2}$, M. Tacca ${ }^{1}$, A. Aguzín ${ }^{3}$, A. Butera ${ }^{4}$, J. Ferrón ${ }^{1,2}$ and C. J. Bonin ${ }^{1,2^{*}}$ \\ ${ }^{1}$ Instituto de Física del Litoral (IFIS-Litoral) - Universidad Nacional del Litoral- CONICET \\ Güemes 3450 - (S3000GLN) Santa Fe - Argentina \\ 2 Departamento de Física, Facultad de Ingeniería Química (FIQ) - Universidad Nacional del Litoral \\ Santiago del Estero 2829 - (S3000AOM) Santa Fe -Argentina. \\ ${ }^{3}$ Facultad de Ingeniería Química (FIQ) - Universidad Nacional del Litoral, Santiago del Estero 2829 \\ (S3000AOM) Santa Fe -Argentina \\ ${ }^{4}$ Instituto de Nanociencia y Nanotecnología, Centro Atómico Bariloche CNEA, Bariloche, Rio \\ Negro, Argentina
}

E-mail: claudio.bonin@santafe-conicet.gov.ar

Received $\mathrm{xxxxxx}$

Accepted for publication $\mathrm{xxxxxx}$

Published xxxxxx

\begin{abstract}
We introduce a novel method aimed to obtain the scale factor between in-plane magnetization components, indispensable for vectorial-Magneto-Optic Kerr Effect (v-MOKE) experiments, convenient for experimental setups where it is difficult or unfeasible to rotate the electromagnet. Unlike the original method, in our proposal the electromagnet (and all other optical components) are fixed, extending in this way the possibility to implement v-MOKE magnetometry. Two films, FePt 9 and $100 \mathrm{~nm}$ thickness were used as sample probes, presenting in-plane magnetic uniaxial anisotropy and in-plane magnetic isotropy, respectively. All experiments were carried out at room temperature and employing a home-made MOKE system. We also introduce a closed mathematical expression for the scale factor linking both magnetization components in terms of experimental and sample dependent parameters.
\end{abstract}

Keywords: vectorial MOKE, surface magnetism, Nanomagnetism, scaling of in-plane magnetic components, magnetic anisotropic film, $\mathrm{FePt}$

\section{Introduction}

Magneto-Optic Kerr Effect (MOKE) is one of the most suitable techniques to study superficial magnetization phenomena due to its limited material penetration $(\sim 20 \mathrm{~nm}$ for most metals [1]). It is widely used in nanomagnetism for its considerable surface sensitivity and relatively simple assemble and operation. The technique is able to sense both, changes in the orientation of electric field polarization plane, and light intensity variations of a monochromatic linearly polarized beam after being reflected by a magnetized surface material. Briefly, the magnetization alters the dielectric tensor of the sample, making it optically anisotropic and hence, modifying the reflection properties of the surface. In a typical experiment, the intensity of the reflected light is acquired while an external magnetic field $\vec{B}$ (that polarizes the sample) 
is cycled. Then, hysteresis cycles of the film are measured since reflected light carries information of the magnetization $\vec{M}=\left(m_{x}, m_{y}, m_{z}\right)$ induced by the field $\vec{B}$. Typically, only one of the three components of the magnetization is measured during a single experiment.

Generally, the technique is mounted in one of the following geometries: longitudinal, transversal or polar [2]. In the longitudinal and transversal configurations, the components $m_{x}$ and $m_{y}$ are detected (magnetization components parallel to the sample plane). The $m_{x}$ and $m_{y}$ components are originated from two different Kerr effects: a change in the state of polarization and a variation in the intensity of the incident light, respectively. Thus, they cannot be directly compared. In addition, as the magnetization of the film does not necessarily follows the direction of the $\vec{B}$ field as this is cycled, the MOKE signal might contain information on the three magnetization components at the same time. Consequently, extracting true in-plane magnetization components is a complex task $[3,4]$. If the film is thin enough, the treatment can be simplified by assuming that the film transversal component $m_{z}$ becomes negligible when compared to the other two magnetization components $\left(m_{z} \approx 0\right)$ [2].

Jiménez et al. [3], proposed a method to simultaneously and quantitatively measure in-plane $m_{x}$ and $m_{y}$ components. To achieve that, they determined a factor that scales both components (crucial for vectorial resolved magnetization reversal studies). This scaling factor is obtained by rotating the electromagnet (they use a small electromagnet), between longitudinal and transversal geometries, and comparing two hysteresis cycles coming from different Kerr effects. In short, they showed that their v-MOKE technique allows studying the dependence of hysteresis and remanence phenomena, critical fields, magnetization reversion processes, determination of the easy and hard axis, domain wall orientation and magnetic anisotropies in a more precise and direct way than with other conventional MOKE setups.

Other groups have proposed diverse methods to measure more than one magnetic components of a film. These techniques are mainly based on measuring hysteresis cycles in different experimental conditions [5-9]: changing the optical components, varying the direction of the magnetic field, or both. All these methods lead to a major practical problem: as the experimental configuration is changed in every single experiment, the whole system must be recalibrated. On the other hand, in the original work reported by Jiménez et al. [3] the experimental setup is only changed once, at the beginning of the experiment, and the scale factor obtained is valid for the remaining measurements.

The method proposed by Jimenez et al. [3] is accurate, easy to implement and appropriate for v-MOKE experiments, however it requires to rotate the electromagnet. Depending on the electromagnet dimensions and weight, in practice, implementing this task could be difficult or directly unfeasible to achieve. Here, we propose a practical solution for these cases allowing them to proceed with Jimenez et al. method, without the need of moving the electromagnet or any other optical component of the MOKE device.

\section{Theoretical description}

In the macroscopic classical description of MOKE experiments, the magnetization breaks the optical symmetry of the sample, making its dielectric tensor anisotropic. If the anisotropy induced by the magnetization of the film is small, some components of the dielectric tensor (non-diagonal elements) will depend linearly with the magnetization (considering up to first order terms). The previous statement constitutes the basis of the mathematical description of the MOKE signal and its connection to the magnetic properties of the film. In the following, we closely follow the theoretical description of ref. [3] and their convenient notation.

The generalized Fresnel coefficients, $r_{s s}, r_{p s}, r_{s p}$ and $r_{s s}$, that relate transversal s-and p-components, can be written in terms of the film magnetization components by using the Jones formalism $[10,11]$ as:

$$
\begin{aligned}
& r_{s s} \approx a ; r_{p p} \approx c+d * m_{y} ; r_{s p} \approx b_{1} * m_{x}+b_{2} * m_{z} ; \\
& r_{p s} \approx-b_{1} * m_{x}+b_{2} * m_{z} .
\end{aligned}
$$

The set of eqs. (1) shows the explicit dependence of the matrix reflection coefficients with the magnetization components $\left(m_{x}, m_{y}\right.$ and $\left.m_{z}\right)$. While the coefficients $a, c, d, b_{1}$ and $b_{2}$ are independent of the magnetization, they depend on several parameters, including refraction indexes of the interface mediums, the wavelength of the incident beam, the incident angle, etc. We will see later that the scale factor depends on $\mathrm{c}$, $\mathrm{d}$ and $\mathrm{b}_{1}$. On the other hand, the magnetization components are dependent on the field strength $B$, ergo $m \lambda(B)$ with $\lambda=x, y, z$. To find all the coefficients involved in (1), the Maxwell equations must be solved at the interface. This calculation is beyond the scope of the present study (see Appendix of ref. [3]).

The final light beam, after interacting with the film surface and the rest of the optical components, is separated by a Wollastone prism into two beams (with intensities $I_{s}$ and $I_{p}$, respectively) that impinge on two photodiodes. The responses of the photodiodes are subtracted and added independently. Finally, they are amplified by a photoamplifier, giving the following outputs (see ref. [3] for more details):

$$
\begin{gathered}
I_{\Delta} \equiv I_{s}-I_{p} \approx 2 I_{0}\left[\operatorname{Re}\left\{b_{1} c^{*}\right\} m_{x}+\operatorname{Re}\left\{b_{2} c^{*}\right\} m_{z}\right. \\
\left.+\operatorname{Re}\left\{b_{1} d^{*}\right\} m_{x} m_{y}+\operatorname{Re}\left\{d b_{2}^{*}\right\} m_{z} m_{y}\right] \\
\begin{aligned}
I_{\Sigma}^{D C} \equiv\left(I_{s}+I_{p}\right)^{D C} \approx I_{0}|c|^{2} \\
I_{\Sigma}^{A C} \equiv\left(I_{s}+I_{p}\right)^{A C} \approx I_{0}\left[2 \operatorname{Re}\left\{d c^{*}\right\} m_{y}+2 \operatorname{Re}\left\{b_{1} b_{2}^{*}\right\} m_{x} m_{z}+\right. \\
\left.+\left|b_{1}\right|^{2} m_{x}{ }^{2}+\left|b_{2}\right|^{2} m_{z}{ }^{2}+|d|^{2} m_{y}{ }^{2}\right]
\end{aligned}
\end{gathered}
$$


where $I_{0} \propto\left|E_{p}^{(i)}\right|^{2}$. Assuming the films are thin enough to consider $m_{z} \approx 0$, and the terms containing squared components of the magnetization are small in comparison with the linear terms, it is seen that eq. (2) is linear with $m_{x}$, while eq. (4) is linear with $m_{y}$. Note that only the linear terms can lead to hysteresis curves. The fact that hysteresis cycles are effectively measured supports the assumptions made. Then, under these assumptions, the signal $I_{\Delta}$ is proportional to the component of the magnetization parallel to the external magnetic field $\left(m_{x} \equiv M_{||}\right)$, while $I_{\Sigma}^{A C}$ is proportional to the magnetization component perpendicular to the magnetic field $\left(m_{y} \equiv M_{\perp}\right)$; both coming from two different Kerr effects:

$$
\begin{gathered}
I_{\Delta}(B, \theta) \approx 2 I_{0} \operatorname{Re}\left\{b_{1} c^{*}\right\} m_{x} \equiv 2 I_{0} \operatorname{Re}\left\{b_{1} c^{*}\right\} M_{||}(B, \theta) G_{\Delta} \\
I_{\Sigma}^{A C}(B, \theta) \approx 2 I_{0} \operatorname{Re}\left\{d c^{*}\right\} m_{y} \equiv 2 I_{0} \operatorname{Re}\left\{d c^{*}\right\} M_{\perp}(B, \theta) G_{\Sigma}^{A C} .
\end{gathered}
$$

Here we explicitly show the dependence of the signals on both the magnetic field $\mathrm{B}$ and the angle of sample rotation $\theta$. The factors $G_{\Delta}$ and $G_{\Sigma}^{A C}$ are the gains of the photoamplifier of each output (generally the same). The $I_{\Sigma}^{D C}(B, \theta)$ signal (with gain $\left.G_{\Sigma}^{D C}\right)$ is used to normalize the above signals, since it accounts for the total reflectivity of the surface, regardless of its magnetization. Then:

$$
\begin{aligned}
& I_{\Delta}^{N}(B, \theta) \approx \frac{2 \operatorname{Re}\left\{b_{1} c^{*}\right\}}{|c|^{2}} M_{\|}(B, \theta) \frac{G_{\Delta}}{G_{\Sigma}^{D C}}=\alpha M_{\|}(B, \theta) ; \\
& \alpha \equiv \frac{2 \operatorname{Re}\left\{b_{1} c^{*}\right\}}{|c|^{2}} \frac{G_{\Delta}}{G_{\Sigma}^{D C}} ; \\
& I_{\Sigma N}^{A C}(B, \theta) \approx \frac{2 R\left\{d c^{*}\right\}}{|c|^{2}} M_{\perp}(B, \theta) \frac{G_{\Sigma}^{A C}}{G_{\Sigma}^{D C}}=\beta M_{\perp}(B, \theta) ; \\
& \beta \equiv \frac{2 \operatorname{Re}\left\{d c^{*}\right\}}{|c|^{2}} \frac{G_{\Sigma}^{A C}}{G_{\Sigma}^{D C}},
\end{aligned}
$$

where $I_{\Delta}^{N}$ and $I_{\Sigma N}^{A C}$ are the corresponding normalized signals. In eqs. (5), the constants $\alpha$ and $\beta$ (which do not depend on the field B) are introduced. These constants do certainly depend on the characteristics of the sample and certain experimental conditions such as the wavelength of the beam and the angle of incidence used.

$$
\begin{aligned}
& \text { From (5), } \\
& \begin{array}{l}
I_{\Sigma N}^{A C}(B, \theta)=\beta M_{\perp}(B, \theta)=\alpha \frac{\beta}{\alpha} M_{\perp}(B, \theta)=\alpha M_{\perp}^{\prime}(B, \theta) ; \\
M_{\perp}(B, \theta)=\frac{\alpha}{\beta} M_{\perp}^{\prime}(B, \theta) \equiv \operatorname{sf} M_{\perp}^{\prime}(B, \theta) .
\end{array}
\end{aligned}
$$

The scale factor $s f \equiv \frac{\alpha}{\beta}=\frac{\operatorname{Re}\left\{b_{1} c^{*}\right\}}{\operatorname{Re}\left\{d c^{*}\right\}} \frac{G_{\Delta}}{G_{\Sigma}^{A C}}$ allows linking the true transverse magnetization $M_{\perp}$ with the measured, not scaled, transverse magnetization $M_{\perp}^{\prime}$. Thus, the proportionality constant $\alpha$ links both magnetizations, $M_{\perp}^{\prime}$ and $M_{\|}$, with the signals $I_{\sum N}^{A C}$ and $I_{\Delta}^{N}$, respectively. As stated above, $\alpha$ and $\beta$ depend on various optical parameter of the sample, then, in principle, the scale factor $s f$ should depend on the sample under study. The practical approach used to determine $s f$ will be detailed later. The factor $\frac{G_{\Delta}}{G_{\Sigma}^{A C}}$ in $s f$ could be determined, for example, covering one of the two photodiode and dividing $I_{\Delta} / I_{\Sigma}^{A C}$.

\section{Experimental method}

A home-built v-MOKE (based on ref. [3]) was used in all the experiments reported.

The hysteresis loops were obtained by cycling a magnetic field $\vec{B}$ (parallel to both the film and the incidence plane) with a $1 \mathrm{~Hz}$ triangular shape pulse $\left(B(t=0)=B_{\max }=\right.$ $+100 \mathrm{mT})$. A maximum B field of $100 \mathrm{mT}$ was sufficient to reach the magnetization saturation in both samples ( $\mathrm{FePt} 9$ and $100 \mathrm{~nm}$ thickness) for all angles. The field B was measured in situ using a transverse Hall probe, placed within the electromagnet gap, in close contact with the sample.

The FePt $9 \mathrm{~nm}$ sample was rotated an angle $\theta$ around an axis parallel to the surface normal from $0^{\circ}$ to $350^{\circ}$ in steps of $10^{\circ}$, while the $100 \mathrm{~nm}$ sample was rotated in the range $0^{\circ}$ to $300^{\circ}$ in steps of $30^{\circ}$, with a fixed $\vec{B}$ field direction. For each $\theta$, the tilt of the sample was manually handled in order to correct defects in our rotation mechanism and the retarder sheet was finely tuned so that $I_{\Delta} \approx 0$ for $M=0$. This handling should not be necessary in case a more sophisticated mechanism is used. Around 300 signals were averaged for each angle in order to improve the signal-to-noise ratio. The beam (generated by a randomly polarized $\mathrm{HeNe}$ laser of $15 \mathrm{~mW}$ at $632 \mathrm{~nm}$ ) with a spot size of $\sim 1 \mathrm{~mm}$ diameter strikes the sample at $\sim 60^{\circ}$ relative to the surface normal, with linear p-polarization (selected by a Glan-Taylor polarizer prism located between the laser source and the sample).

\section{Samples}

The FePt $9 \mathrm{~nm}$ thick film presents in-plane uniaxial anisotropy, with uniform magnetized domains, giving a square hysteresis cycles when the $\vec{B}$ field is cycled in the direction of the easy axis, parallel to the film. However, if the field $\vec{B}$ is applied perpendicular to the $9 \mathrm{~nm}$ film, it reveals a relatively weak uniaxial anisotropy. This anisotropy is responsible for the change in the domain structure when the FePt film thickness exceeds a critical value $(\sim 30 \mathrm{~nm})$. Then, for the 9 $\mathrm{nm}$ thick film, the magnetization reverses its direction mostly parallel to the plane of the film $\left(m_{z} \approx 0\right)$; but the $100 \mathrm{~nm}$ thick film (thicker than the critical thickness) reverses its magnetization in a two-step process, typical of systems that show stripe-like magnetic domains [12-14]. In this case, a small component of the magnetization, transverse to the plane of the film, would appears (i.e. $m_{z} \neq 0$ ), alternating between stripe-like regions with an 'up' and 'down' directions; giving then, an almost net zero contribution to the signal due to the large sensing area of the beam spot.

\section{Data preprocessing.}


The signals $I_{\Sigma}^{D C}$ (DC component of the sum of the signals of each photodiode) were replaced by constant functions equal to the mean values of each data distribution in order to obtain a set of free of noise reference signals $I_{0}$. These functions were used to divide each signal acquired, coming from the AC component of the sum $I_{\Sigma}^{A C}$, and the difference of the signals of both photodiodes $I_{\Delta}$, respectively (i.e., $I_{\Delta} / I_{\Sigma}^{D C} \propto M_{\|}$and $I_{\Sigma}^{A C} / I_{\Sigma}^{D C} \propto M_{\perp}^{\prime}$, with the same proportionality constant). In this way, possible variations in the incident beam intensities during the hysteresis cycle are corrected. Then, the signals were averaged and corrected by an offset for each angle, so that the hysteresis cycle is centered around $(M, B)=(0,0)$. The final hysteresis curves (see Fig.1) are then obtained from the time evolutions of the signals $I_{\Delta} / I_{\Sigma}^{D C}$ and $I_{\Sigma}^{A C} / I_{\Sigma}^{D C}$ vs. the intensity of the applied magnetic field, $B$.

\section{Results and Discussion}

\section{Hysteresis cycles}

In Figs. 1(a) and (b) we show the hysteresis curves of the parallel magnetization $M_{\|}$and transverse component $M_{\perp}^{\prime}$ respectively, for four rotation angles of the $9 \mathrm{~nm}$ FePt sample.

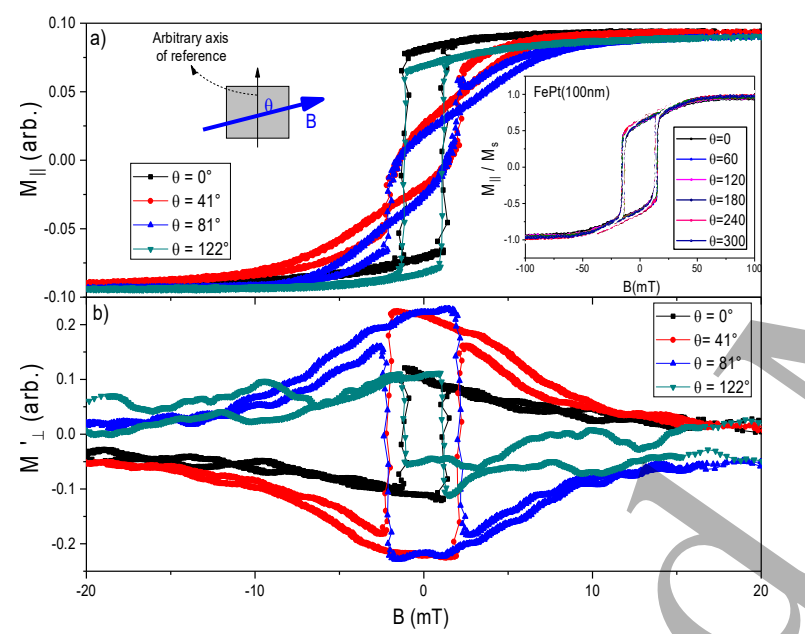

Figure 1. Hysteresis cycles of FePt $(9 \mathrm{~nm})$ sample, corresponding to $M_{\| !}$(a) and $M^{\prime}{ }_{\perp}$ (b) for four different rotation angles. Signals are not normalized. Inset of figure 1(a): Hysteresis cycles of the sample FePt $(100 \mathrm{~nm})$, corresponding to $M_{\|}$and for several rotation angles. The signals are normalized to the saturation $M_{s}$ values.

The graphs clearly show different hysteresis cycles for these magnetization components and different y-scales due to different Kerr effects. Data of Fig. 1(a) are reduced by the scale factor with respect to data of Fig. 1(b). Both provide complementary information that can be used to describe the magnetization reversion processes of the film when they are correctly scaled. In addition, the poorer signal-to-noise ratio obtained in Fig. 1(b) indicates that the transverse component signal is much weaker than that of the parallel magnetization component.

In the inset of Fig. 1(a) we show the hysteresis curves obtained for the parallel magnetization $M_{\|}$of the FePt $100 \mathrm{~nm}$ sample. For this sample, the transverse component of the magnetization is zero (under detection limits). Additionally, this curves show no evidence of magnetic anisotropy in the plane of the film.

\section{Scale factor between $M_{\|}$and $M_{\perp}^{\prime}$}

Consider that the experiment is performed with the $\vec{B}$ field parallel to an easy axis (e.a.) of the sample and that true inplane magnetization components are complementary. Then an almost square hysteresis cycle is obtained and we have:

$$
M_{s}^{2}=M_{s \|}^{2}+M_{s \perp}^{2}=M_{r \|}^{2}+M_{r \perp}^{2}
$$

where subscripts $r$ and $s$ indicate remanence and saturation (at $\mathrm{B}_{\max }$ ), respectively. Remanent magnetizations, both for the parallel and perpendicular components, are determined from the hysteresis curves at the field value $\mathrm{B}=0$ for each angle $\theta$, as the average value between the positive and negative remanence values (as suggested in ref. [3], i.e. $M_{r \|}=$ $\frac{M_{\|}^{+}(B=0)-M_{\|}^{-}(B=0)}{2}$. An analogous calculation is performed for the transverse component.

From the measured signals (Fig. 1) and the set of eqs. (5) and (6), we obtain:

$$
\begin{aligned}
& I_{\Delta}^{N}\left(B_{\text {max }}, \theta\right)=\alpha M_{\|}\left(B_{\text {max }}, \theta\right) \equiv \alpha M_{s \|}(\theta) ; \\
& I_{\Sigma N}^{A C}\left(B_{\max }, \theta\right)=\alpha M_{\perp}^{\prime}\left(B_{\max }, \theta\right) \\
& \equiv \beta M_{s \perp}(\theta) \equiv \alpha M_{s \perp}^{\prime}(\theta) ; \\
& =\frac{\alpha}{s f} M_{\perp}(0, \theta) \equiv \frac{\alpha}{s f} M_{r \perp}(\theta) .
\end{aligned}
$$

Inserting eqs. (8) and (9) in eq. (7):

$$
\begin{gathered}
\frac{\left(I_{\Delta}^{N}\left(B_{\text {max }}, \theta\right)\right)^{2}}{\alpha^{2}}+\frac{\left(I_{\sum N}^{A C}\left(B_{\text {max }}, \theta\right)\right)^{2}}{\beta^{2}} \\
=\frac{\left(I_{\Delta}^{N}(0, \theta)\right)^{2}}{\alpha^{2}}+s f^{2} \frac{\left(I_{\sum N}^{A C}(0, \theta)\right)^{2}}{\alpha^{2}} \\
1=\frac{\left(I_{\Delta}^{N}(0, \theta)\right)^{2}}{\left(I_{\Delta}^{N}\left(B_{\max }, \theta\right)\right)^{2}}+s f^{2}\left[\frac{\left(I_{\sum N}^{A C}(0, \theta)\right)^{2}}{\left(I_{\Delta}^{N}\left(B_{\max }, \theta\right)\right)^{2}}-\frac{\left(I_{\sum N}^{A C}\left(B_{\max }, \theta\right)\right)^{2}}{\left(I_{\Delta}^{N}\left(B_{\max }, \theta\right)\right)^{2}}\right] \\
=\frac{M_{r \| \mid}^{2}(\theta)}{M_{S||}^{2}(\theta)}+s f^{2}\left[\frac{\left(M_{r \perp}^{\prime}(\theta)\right)^{2}}{M_{S||}^{2}(\theta)}-\frac{\left(M_{S \perp}^{\prime}(\theta)\right)^{2}}{M_{S \| \mid}^{2}(\theta)}\right] .
\end{gathered}
$$

From the data (see Fig. 1) it can be seen that $M_{s \perp}^{\prime}(\theta) \ll$ $M_{r \perp}^{\prime}(\theta)$, and Eq. (10) can be approximated as:

$$
1 \approx \frac{M_{r||}^{2}(\theta)}{M_{S||}^{2}(\theta)}+s f^{2} \frac{\left(M_{r \perp}^{\prime}(\theta)\right)^{2}}{M_{S \|}^{2}(\theta)} .
$$

Eq. (11) implies that, from a linear fit of $\frac{M_{r \| \mid}^{2}(\theta)}{M_{S||}^{2}(\theta)}$ vs. $\frac{\left(M_{r \perp}^{\prime}(\theta)\right)^{2}}{M_{S||}^{2}(\theta)}$, 
measured for different orientations of the sample, the scaling factor can be obtained. Fig. 2 precisely shows this dependence and the corresponding linear fit. The linear dependence of the data obtained supports the assumptions made in our method.

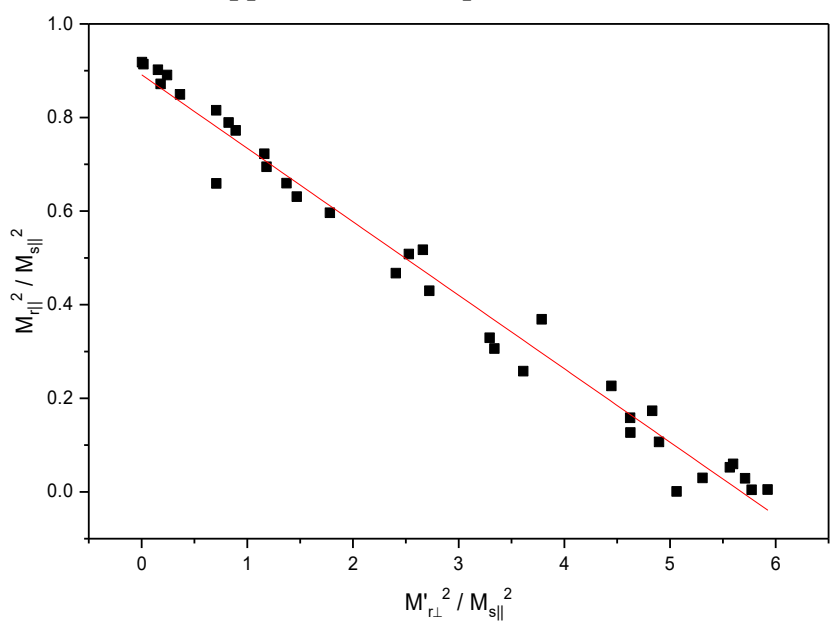

Figure 2: $\frac{M_{r||}^{2}(\theta)}{M_{S||}^{2}(\theta)}$ Vs. $\frac{\left(M_{r \perp}^{\prime}(\theta)\right)^{2}}{M_{S||}^{2}(\theta)}$ plot aimed to obtain the scale factor, $s f$. The solid line corresponds to the linear fit of data. The slope is the square of the scale factor.

Each experimental point in Fig. 2 corresponds to an angular position $(\theta=0$ arbitrary chosen) of the sample relative to the field $\vec{B}$. The fitting parameters obtained with their statistical errors are $(0.89 \pm 0.01)$ for the intercept and $(-0.157 \pm 0.003)$ for the slope, with a correlation factor $R \approx 0.98$. From here, the scaling factor can be obtained as the square root of the slope:

$$
s f=0.40 \pm 0.01 \text {. }
$$

The value of the scale factor obtained here is strikingly equal to that reported in ref. [3]. However, the sample used and some of the experimental conditions employed here are rather different than that of [3]. We will come back to this issue in the Conclusions section.

\section{Angular dependence of hysteresis parameters}

In Fig. 3(a) (upper panel) hysteresis loops are shown for the parallel and transversal magnetizations of the FePt $(9 \mathrm{~nm})$ sample, normalized to the saturation $M_{s}$ value and scaled by the $s f$ factor determined above. These loops correspond to three rotation angles of the sample relative to the direction of the external field $\vec{B}$.

The angles were selected to show the variety of hysteresis curves. These diverse magnetization reversal processes presented by the FePt $(9 \mathrm{~nm})$ become more evident (as suggested in ref. [3]) from the polar plot representation shown in Fig. 3(b) (lower panel). From these plots, it is possible to quickly identify the easy axis of magnetization, which is close to the angle $\theta=152^{\circ}$. Here, the magnetization is always aligned with the external field $\vec{B}$, i.e., $M_{\perp}=0 . M_{\|} / M_{S}$ falls on the unit circle during its inversion from the value $B_{\max }$ up to the value of the coercive field $B_{c}$, at which the magnetization is reversed abruptly and irreversibly, almost to its minimum value, where the film is magnetically saturated in the opposite direction. Magnetic domains are easily reversed for this direction, contrary to what occurs for other angles, where the magnetization does not strictly aligns with the field and a transversal in-plane component appears when the field is $\operatorname{cycled}\left(M_{\perp} / \mathrm{M}_{\mathrm{s}} \neq 0\right)$.
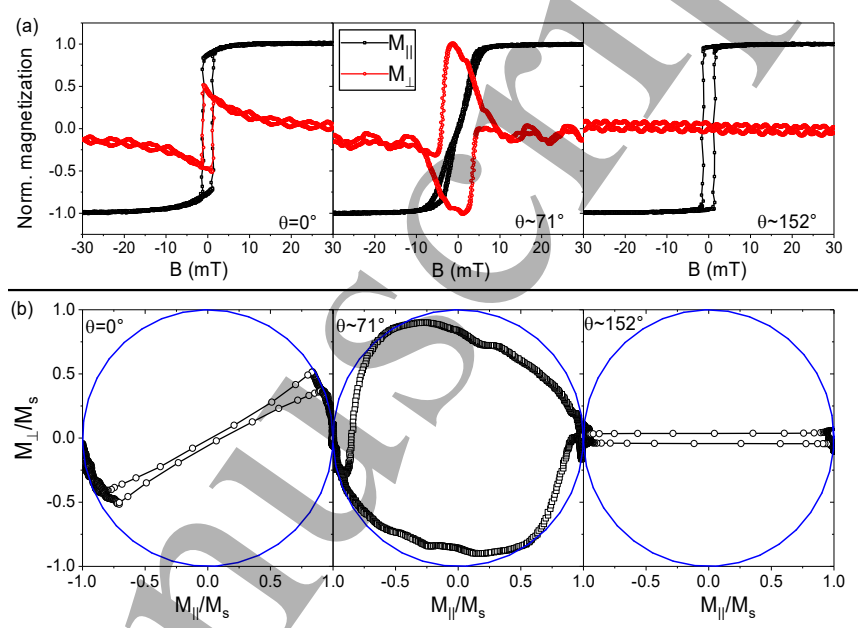

Figure 3: (a): Hysteresis loops of the parallel (black) and transversal (red) magnetization components for the $\mathrm{FePt}(9 \mathrm{~nm})$ film, scaled and normalized to the saturation $M_{S}$ value, for three different angles; (b) the corresponding polar plot representations. Here, the different magnetization inversion mechanisms can be clearly identified.

In Fig. 4 the remanence magnetization values (normalized and scaled) are plotted as a function of the sample rotation angle $\theta$, both for the parallel and perpendicular components ( 9 $\mathrm{nm}$ and $100 \mathrm{~nm}$ FePt are compared).

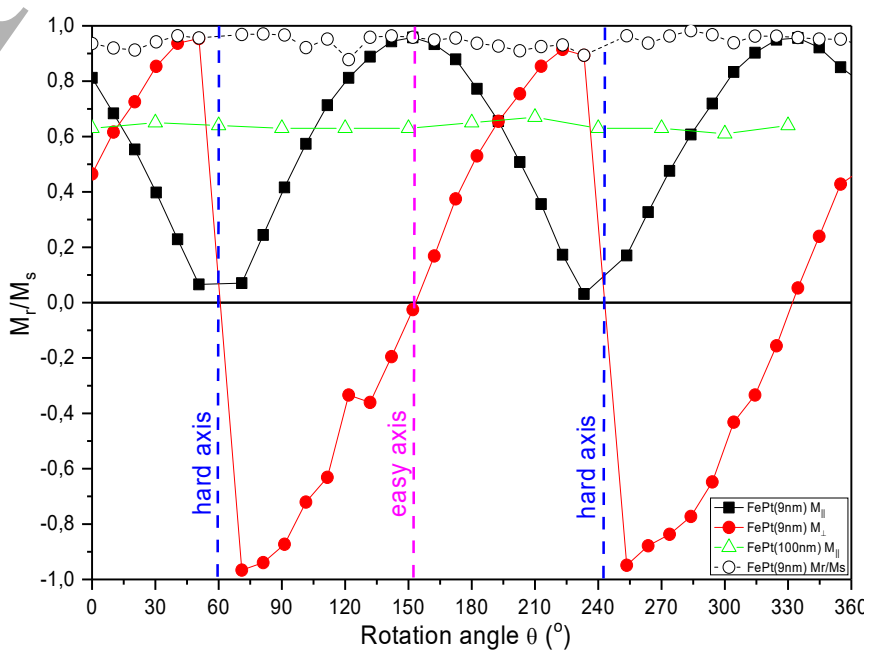

Figure 4: Angular dependence of normalized and scaled remanent magnetization components for both FePt films, 9 and $100 \mathrm{~nm}$ thickness. $M_{r} / M_{s}$ (open circles) is also shown for the $9 \mathrm{~nm}$ film. Dashed line indicates the respective easy (magenta) and hard (blue) axes.

The $9 \mathrm{~nm}$ FePt sample presents a clear uniaxial magnetic anisotropy, evidenced by the abrupt changes of the transverse component of the magnetization at the angles near $60^{\circ}$ and 
$240^{\circ}\left(180^{\circ}+60^{\circ}\right)$, and a smooth zero-crossing at an angle of $150^{\circ}$. Note that, in contrast to conventional MOKE arrangement, with v-MOKE the identification of the hard and easy axes is direct, coming from two different sources: i) the parallel component of the remanence magnetization reachs its minimum value for angles close to the hard axis (h.a.), and reachs its maximum value for the easy axis (e.a.) and, ii) the transverse magnetization presents an step behavior for the h.a. and continuosly (smoothly) goes to zero near the e.a. It is also important to highlight the $180^{\circ}$ rotational symmetry showed by both components of the magnetization, indicating a clear uniaxial magnetic anisotropy. Contrary to what happens with the thinnest FePt film, the remanence magnetization in the 100 $\mathrm{nm}$ sample does not depend on the rotation angle, indicating a clear magnetic isotropy of the sample in the plane of the film.

\section{Conclusions}

We proposed a solution to the problem of implementing vMOKE when it is difficult or impractical to rotate the electromagnet. With this method it is possible to determine the scale factor between in plane magnetization components, essential to proceed with the analysis of data originally described in ref. [3] for vectorial resolved magnetization reversion experiments. An analytical expression was found for this factor in terms of different coefficients that depend both on different physical properties of the sample (refractive index, reflectivity) and experimental parameters (wavelength of the laser source, angle of incidence). The value obtained $(0.40 \pm 0.01)$ surprisingly coincide with that found by other autors, obtained in a different way and using a different sample film. This fact encourage further research focused to unravel the physical ingredients that determine the parameters $b_{1}, d$ and $c$ on which the scale factor depends. At this point, it is only speculated that the wavelength of the laser beam and the angle of incidence are the most determinant parameters in the scale factor. This proposal is based on that these parameters were the same in both studies. If this is correct, the procedure described here would be even more useful since the scale factor would be valid for any sample studied.

The reliability of the introduced method was proved by the results obtained and its contrast with those presviously reported: i) the $9 \mathrm{~nm} \mathrm{FePt} \mathrm{sample} \mathrm{shows} \mathrm{a} \mathrm{hard} \mathrm{axis} \mathrm{shifted}$ $90^{\circ}$ with respect to the direction of the easy axis, while the 100 $\mathrm{nm}$ film does not show any evidence of in-plane anisotropy, ii) the coercitive field measured were $1.5 \mathrm{mT}$ and $15 \mathrm{mT}$ for the thiner and thicker films, irrespective of the rotation angle and iii) in the easy axis direction, the remanence magnetization of the $9 \mathrm{~nm}$ film is higher than that of the 100 $\mathrm{nm}$ film sample. These three results fully agree with those reported in [12-14].

Finally, we showed that the v-MOKE setup allows identifying the presence of magnetic anisotropies, determining the easy and hard magnetic axes in a precise and direct way.

\section{Acknowledgements}

We acknowledge to CONICET for financial support. C. J. B. would like to thank Julio Camarero for his insightful comments and advices concerning the v-MOKE setup.

\section{References}

[1] Bland JAC, Padgett MJ, Butcher RJ, Bett N 1989 An intensitystabilised He-Ne laser for measuring small magneto-optic Kerr rotations from thin ferromagnetic films J. Phys. E Sci. Instrum. 22 308-12.

[2] Qiu ZQ, Bader SD 2000 Surface magneto-optic Kerr effect Rev Sci. Instrum. 71 1243-55.

[3] Jiménez E, Mikuszeit N, Cuñado JLF, Perna P, Pedrosa J, Maccariello D, Rodrigo C, Niño MA, Bollero A, Camarero J, 2014 Vectorial Kerr magnetometer for simultaneous and quantitative measurements of the in-plane magnetization components Rev. Sci. Instrum. 85053904.

[4] Soldatov IV, Schäfer R 2017 Selective sensitivity in Kerr microscopy Rev. Sci. Instrum. 88073701.

[5] Vavassori P 2000 Polarization modulation technique for magneto-optical quantitative vector magnetometry Appl. Phys. Lett. 77 1605-7.

[6] Morales R, Martín JI, Vélez M, Alameda JM 2004 Magnetiza tion reversal measurements in mesoscopic amorphous magnets by magneto-optical Kerr effect Eur. Phys. J. B 40 463-70.

[7] Zhan Q-f, Vandezande S, Temst K, Van Haesendonck C 2009 Magnetic anisotropies of epitaxial $\mathrm{Fe} / \mathrm{MgO}(001)$ films with varying thickness and grown under different conditions New $J$. Phys. 11063003.

[8] Kuschel T, Bardenhagen H, Wilkens H, Schubert R, Hamrle J, Pišstora J, Wollschläger J 2011 Vectorial magnetometry using magnetooptic Kerr effect including first- and second-order contributions for thin ferromagnetic films J. Phys. D Appl. Phys. 44265003.

[9] Teixeira JM, Lusche R, Ventura J, Fermento R, Carpinteiro F, Araujo JP, Sousa JB, Cardoso S, Freitas PP 2011 Versatile, high sensitivity, and automatized angular dependent vectorial Kerr magnetometer for the analysis of nanostructured materials Rev. Sci. Instrum. 82043902.

[10] Azzam RMA, Bashara NM. Ellipsometry and polarized light. Amsterdam; New York: North-Holland : Sole distributors for the USA and Canada, Elsevier Science Pub. Co.; 1987.

[11] Florczak JM, Dahlberg ED 1990 Detecting two magnetization components by the magneto-optical Kerr effect J. Appl. Phys. $677520-5$.

[12] Mansilla MV, Gomez J, Butera A 2008 Ferromagnetic Resonance of Disordered FePt Thin Films IEEE Trans. Magn. 44 2883-6.

[13] Sallica Leva E, Valente RC, Martínez Tabares F, Vásquez Mansilla M, Roshdestwensky S, Butera A 2010 Magnetic domain crossover in FePt thin films Phys. Rev. B 82144410.

[14] Burgos E, Sallica Leva E, Gómez J, Martínez Tabares F, Vásquez Mansilla M, Butera A 2011 Surface pinning in ferromagnetic films with perpendicular anisotropy Phys. Rev. B 83174417. 